\title{
El derecho a votar desde el extranjero
}

\author{
Pablo Marshall Barberán*
}

\begin{abstract}
RESUMEN
Este trabajo presenta ciertos elementos, principalmente tomados del derecho comparado y de la teoría politica, que pueden hacer más inteligible la discusión sobre el sufragio desde el extranjero en el sistema político chileno. La primera parte de este trabajo muestra la importancia de discutir su fundamento, mediante la aclaración de algunas de sus premisas filosóficas. La segunda parte del trabajo examina el proyecto de ley de reforma constitucional que fuera rechazado en el Congreso, concluyendo que lo afectaban problemas significativos desde una perspectiva teórica. Finalmente, en la tercera parte del trabajo se consideran en concreto los elementos que una legislación sobre el voto desde el extranjero debe contemplar.
\end{abstract}

Voto desde el extranjero - derecho a voto - democracia

The right to vote from abroad

\begin{abstract}
This paper presents certain elements, mainly taken from comparative law and political theory, which can make more intelligible the discussion on the vote from abroad in the Chilean political system. The first part of this work shows the importance of discussing its foundation by clarifying some of its philosophical premises. The second part of this paper examines the constitutional reform bill that was rejected by Congress, concluding that it was affected by significant theoretical problems. Finally, the third part of the paper considers the particular elements that legislation on voting from abroad should consider.
\end{abstract}

Vote from abroad - right to vote - democracy

* Abogado, Profesor de Derecho Constitucional en la Facultad de Ciencias Jurídicas y Sociales de la Universidad Austral de Chile, Valdivia, Chile. Pablo.marshall@gmail.com

Artículo recibido el $1^{\circ}$ de agosto de 2011 y aceptado para su publicación por el Comité Editorial el 28 de octubre de 2011. 


\section{INTRODUCCIÓN}

$\mathrm{R}$

ecientemente se ha desatado una nueva polémica por la añeja propuesta de extender la posibilidad de votar a los ciudadanos residentes en el exterior. La cuestión, que ahora fue planteada por la Alianza y rechazada por la Concertación, requiere del análisis tanto de elementos estratégicos como de principio. Los argumentos estratégicos son, por supuesto, la principal preocupación de los partidos políticos. Averiguar cómo va a afectar una reforma tal al flujo de votos de una u otra coalición parece ser la cuestión más apremiante. Junto a ella, está la preocupación de estrategias de mitigación del daño probable o, más derechamente, la elaboración de patrones de atribución del voto que favorezcan o al menos no perjudiquen las aspiraciones electorales del respectivo sector.

Por supuesto que estos elementos estratégicos están vestidos con una argumentación de principio. Argumentos sobre la democratización y la justicia componen una madeja indescifrable para quienes quieren observar el forcejeo parlamentario. Pero, contrariamente a lo que se pueda pensar, el asunto es efectivamente gobernado por consideraciones más profundas. Que la motivación de los actores políticos que promueven la reforma no sea primordialmente aplicar dichos principios, o que tal motivación tienda a ser opacada por sus consideraciones estratégicas, no hace que en el trasfondo del discurso sobre la extensión de los derechos de ciudadanía no esté actuando una fuerza que aspira a la democratización y a la justicia. En ese entendido, no es extraño que la mitad de los países a lo largo del mundo permitan a sus ciudadanos, al menos parcialmente, votar desde el extranjero ${ }^{1}$.

Sin embargo, la democracia y la justicia son fácilmente malentendidas, y la implementación de sus premisas en la práctica suele ser causa de desencuentros duraderos y profundos antagonismos. Si lo que se quiere hacer extendiendo el voto hasta el extranjero es realizar una acción basada en consideraciones de principio, corresponde entender cómo es que dicha medida puede satisfacer los principios esgrimidos y analizar si no hay otros principios importantes a considerar que pueden resultar perjudicados por una reforma de estas características.

Como ya se mencionó, la situación que vive nuestro país no es ajena a la experiencia comparada. Los cálculos electorales y las consideraciones pragmáticas de todo tipo han sido un elemento común en la implementación de medidas similares en otros países. Por ejemplo, el otorgamiento de voto a los militares que luchaban por Canadá en la Primera Guerra Mundial pretendió contrapesar el recién otorgado voto femenino durante dicho periodo. En Austria, el año 1990 el partido conservador permitió el voto en el extranjero,

\footnotetext{
${ }^{1}$ Braun, N. y Gratschew, M., “Introduction” en Ellis, A., Navarro, C., Morales, I. y Wall, A. (editores), Voting from abroad: the International IDEA handbook, Stockholm, International Institute for Democracy and Electoral Assistance / The Federal Electoral Institute of Mexico, 2007, p. 3.
} 
mientras el partido socialdemócrata estableció el voto para los residentes no ciudadanos en 2003. Por supuesto, todo ello fundado en cálculos electorales ${ }^{2}$.

Este trabajo revisa algunas importantes consideraciones teóricas respecto del voto desde el extranjero. Luego analiza críticamente las características y el alcance del proyecto de ley que pretendía implementarlo en Chile. Finalmente considera algunos de los problemas que un proyecto de este tipo debe enfrentar para dar cuenta adecuadamente de su finalidad.

\section{Fundamentos DEL VOTO EN EL EXTRANJERO}

\section{Contexto}

La pregunta sobre el voto en el extranjero cobra especial sentido en el contexto de un mundo globalizado. Hay dos factores que llevan a concluir lo anterior. El primero es relativo a la migración internacional. En nivel de migración alcanzado a finales del siglo XX es incomparablemente superior con cualquier otro en la historia ${ }^{3}$. Éste ha llevado a que una inmensa cantidad de personas vivan fuera del país donde nacieron o al que culturalmente pertenecen. Tanto por razones educacionales, laborales o familiares, millones de personas se desplazan cada año, tanto para establecerse permanentemente en el extranjero como para realizar estadías de corto o mediano plazo fuera de su país. Lo que esta realidad ha generado es el derrumbe de la antigua concepción geográfica de la ciudadanía. Ello ha involucrado el traslape de comunidades nacionales con Estados que conservan jurisdicciones totalmente separadas; la proliferación de fenómenos tales como duplicidad de ciudadanías; la asignación de estatutos intermedios de ciudadanía y residencia, y la combinación de los estatus de residente permanente sin ciudadanía y de ciudadano ausente permanentemente de su país de origen para describir una sola persona. Se ha generado toda una nueva constelación de situaciones que, se ha sugerido, deben hacer repensar las tradicionales categorías de ciudadanía y sus derechos asociados ${ }^{4}$.

El segundo elemento es relativo a la evolución del derecho a voto. Si bien sólo a mediados del siglo pasado el sufragio universal se convirtió en una realidad en el mundo occidental, hoy parece haber cierto acuerdo respecto a que una democracia que se precie de ser tal requiere contar con un universo de votantes que tienda a la universalidad.

${ }^{2}$ Ellis, A., "The history and politics of external voting”, en Ellis, A., Navarro, C., Morales, I. y Wall, A. (editores), Voting from abroad: the International IDEA handbook, Stockholm, International Institute for Democracy and Electoral Assistance / The Federal Electoral Institute of Mexico, 2007, pp. 42 y ss; Bauböck, R., "Expansive Citizenship-Voting beyond Territory and Membership", en Political Science and Politics, Vol. 38, n. 4, 2005, p. 685 .

${ }^{3}$ Véase el reporte de la Comisión Global sobre Migración Internacional (2005). Disponible en http:// www.gcim.org/attachements/gcim-complete-report-2005.pdf

${ }^{4}$ Bauböck, R., "Stakeholder citizenship and transnational political participation: A normative evaluation of external voting”, en Fordham Law Review, Vol. 75, 2006, pp. 2394-6. 
El principio de una persona un voto aparece esencialmente incontrovertido 5 . Lo que sí parece ser una novedad en relación con el voto es que las comunicaciones han sido llevadas a un nivel de sofisticación tal, especialmente en el nivel de la tecnología, que no se ven impedimentos de orden técnico para la implementación de un sistema de votación a distancia. Y no sólo eso, la tecnología de las comunicaciones permite hoy en día mantener comunicadas a personas que están dispersas a través del mundo, actualizándolas con la realidad de sus países de origen, dando cuenta así de la tradicional objeción de la brecha existente entre los asuntos que afectan al país y la capacidad de los residentes en el extranjero para actuar responsable e informadamente ${ }^{6}$.

Con todo, en la práctica el voto desde el extranjero se ha puesto paulatinamente en la agenda política de los Estados, principalmente desde la caída del comunismo y la consecuente búsqueda de diseños electorales para los países que se incorporaron al sistema democrático a fines de la década de los ochentas y comienzos de los noventas ${ }^{7}$. La experiencia de transición de nuestro país tambien permite entender cómo la pregunta del voto desde el extranjero se planteó, desde el retorno a la democracia en 1990, como parte de ese momento democratizador.

No obstante la importancia de esos elementos, que podrían considerarse detonantes del auge de este tipo de regulación, ella tiene particulares y diversas causas en los diferentes países donde ha sido implementada. Una rápida revisión de dichas causas lleva a concluir que las razones para instaurar el voto desde el extranjero pueden variar conforme al contexto político e histórico. Así, esto puede deberse a razones como: (i) la voluntad de permitir la participación de personas que han sido forzadas por la guerra u otros deberes con el Estado a estar lejos de su país (como sucedió en el Reino Unido durante el primera guerra, o Francia durante la segunda guerra); (ii) la pretensión de reparar e incluir simbólicamente, en el contexto de transiciones a la democracia, a quienes han sido expulsados violentamente por razones políticas por un régimen dictatorial (como sucedió en España en 1978); (iii) la respuesta a una demanda constante por parte de los ciudadanos que viven en el extranjero; o (iv) la genérica voluntad estatal de mantener un lazo de unión con quienes emigran del país, a lo cual puede subyacer tanto una cierta comprensión etnocéntrica de la ciudadanía como el interés de mantener a potenciales contribuyentes vinculados por cierto vínculo de lealtad ${ }^{8}$.

${ }^{5}$ Lo que está resumido en la expresión una persona un voto es la idea de que cada persona debe tener derecho igual a votar y que todos los votos deben ser contados por igual, Dahl, R., On Political Equality, New Haven, Yale University Press, 2006, p. 9. De forma crítica, pero desde una mirada superficialmente aritmética, véase Hayden, G.M., "False Promise of One Person, One Vote”, en Michigan Law Review, Vol. 102, 2003.

${ }^{6}$ Bauböck, op. cit., n. 4, p. 2429.

${ }^{7}$ Braun et al., op. cit., n. 1, p. 3; Bauböck, op. cit., n. 2, p. 686; Nohlen, D. y Grotz, F., "External voting: legal framework and an overview of electoral legislation", en Boletín Mexicano de Derecho Comparado, Vol. 99, 2000, p. 1116.

${ }^{8}$ Ellis, op. cit., n. 2, p. 44; Nohlen y Grotz, op. cit., n. 7, p. 1123; Bauböck, op. cit., n. 2, pp. 683-4; Collyer, M. y Vathi, Z., "Patterns of Extra-territorial Voting”, Working Paper T22, Sussex Centre for Migration Research, October, 2007, p. 8. 
No obstante ser todas estas razones pertinentes en la discusión sobre el voto desde el extranjero en Chile, el análisis que sigue se organizará de una manera diferente. Primero, es esencial entender de qué se habla cuando se lo hace sobre el sufragio desde el extranjero. Sólo sobre esa línea se podrán revisar algunas ideas generales sobre ciudadanía y democracia que están en la base de la discusión filosófica sobre su procedencia. Luego se revisarán algunas de las principales propuestas teóricas para justificar o desechar el voto desde el extranjero.

\section{Una reformulación de las preguntas}

La discusión sobre el voto en el extranjero tiende a formalizarse en extremo, focalizándose en la cuestión de si los ciudadanos pueden o no votar desde fuera del país. Por ejemplo, al tratar el problema Nohlen considera que la pregunta sobre el voto desde el extranjero consiste en determinar si existe "un procedimiento de votación realizado en el territorio de un país extranjero por ciudadanos que viven fuera de su país de origen"9. Este enfoque parte de una premisa que es posible discutir aquí, que es que todos los ciudadanos que se encuentran fuera del país tienen el mismo estatus en relación a la posibilidad de ejercer sus derechos políticos.

La discusión sobre esta materia puede enriquecerse substancialmente si se distinguen las dos siguientes preguntas: (1) ¿debe existir un sistema de votación que permita a los ciudadanos ausentes del país el día de la elección ejercer su derecho a voto?; y (2) ¿deben los ciudadanos no residentes en el país estar habilitados para votar? y si ese es el caso, ¿bajo qué circunstancias? En otras palabras, la distinción que se propone es entre procedimiento de votación desde el extranjero y la titularidad del derecho a voto en el extranjero.

Como se puede observar, la respuesta a estas preguntas puede ser distinta. Es cierto que esto puede parecer una trampa argumentativa, dado que es difícil responder negativamente a la primera pregunta si se responde positivamente a la segunda. Sin embargo, como puede concluirse tras una breve revisión de las legislaciones comparadas, tanto los principios que hay detrás de las respuestas a estas preguntas como los sistemas para implementarlos no son cuestión de todo o nada, sino que admiten distinciones y graduaciones que deben ser cuidadosamente consideradas ${ }^{10}$.

La distinción entre procedimiento y titularidad deja en evidencia la principal preocupación de un punto de vista filosófico, llamado así para oponerlo al enfoque formalista que entiende la pregunta por el voto desde el extranjero exclusivamente en términos de procedimiento. Este enfoque consiste en diferenciar a los ciudadanos que viven permanentemente en su país de origen (en adelante, ciudadanos residentes) de aquellos individuos que no obstante tener el estatus de ciudadano no tienen residencia

${ }^{9}$ Nohlen y Grotz, op. cit., n. 7, p. 1119.

${ }^{10}$ Navarro, C., Morales, I., Gratschew, M., "External voting: a comparative overview", en Ellis, A., Navarro, C., Morales, I. y Wall, A. (editores), Voting from abroad: the International IDEA handbook, Stockholm, International Institute for Democracy and Electoral Assistance / The Federal Electoral Institute of Mexico, 2007. 
en su país de ciudadanía (en adelante, expatriados). Bajo esta distinción, los ciudadanos que se encuentran accidentalmente en el extranjero pertenecen a la primera categoría, por lo que la respuesta a la pregunta sobre el procedimiento podría ser respondida tratando a este grupo de forma diferente a los expatriados ${ }^{11}$.

\section{Dos eslóganes usuales}

Antes de considerar más sustantivamente el problema de quiénes deben tener derecho a sufragio, deben descartarse dos argumentos que son usualmente esgrimidos por los defensores del voto desde el extranjero. El problema con estos argumentos es que son retóricas poderosas pero teóricamente débiles respecto de razones para la inclusión de los expatriados.

El primer caso es el eslogan de la democracia. Se ha dicho que el voto desde el extranjero favorece la democratización. ¿Qué es lo que esto significa? Por supuesto que no es nada claro. Para empezar, se puede constatar que no hay correlación alguna entre el nivel de democratización de un Estado y la introducción del sufragio desde el extranjero. Para ello se pueden observar algunos ejemplos, entre los ex países del bloque comunista, de democracias maduras que no lo aplican (República Checa o Hungría) y países con regímenes autoritarios que sí la aplican (Kazajstán o Bielorrusia). La introducción del sufragio desde el extranjero se debe, como se mencionó, antes a peculiaridades nacionales que al efecto de la oleada de democratización que afectara al mundo en los años noventa ${ }^{12}$. No obstante, conceder que democratización y voto en el extranjero no van necesariamente de la mano, no fuerza a concluir que un análisis del significado de la democracia no pueda ayudar a entender, ahora sí desde un punto de vista normativo, si esta forma de sufragio es procedente. La siguiente sección está destinada particularmente a evaluar la relación entre democracia y el voto de los expatriados.

El segundo eslogan a analizar es el de los derechos humanos. Desde el punto de vista de los derechos humanos, el argumento a favor del sufragio desde el extranjero puede ser planteado considerando que las declaraciones internacionales de derechos humanos consideran el derecho inalienable a un sufragio universal e igualitario. A ambas características pueden ser imputadas la necesidad de satisfacer el derecho de los ciudadanos que residen en el exterior. Sin embargo, concretamente, la institución es mencionada solamente en el Convenio Internacional para la Protección de los Trabajadores Migrantes, cuyo artículo 41 sostiene

Los trabajadores migratorios y sus familiares tendrán derecho a participar en los asuntos públicos de su Estado de origen y a votar y ser elegidos en elecciones celebradas en ese Estado, de conformidad con su legislación.

\footnotetext{
${ }^{11}$ Bauböck, op. cit., n. 4, pp. 2398-9.

${ }^{12}$ Nohlen y Grotz, op. cit., n. 7, p. 1121.
} 
Los Estados de que se trate facilitarán, según corresponda y de conformidad con su legislación, el ejercicio de esos derechos.

Si bien este convenio ha sido ratificado por Chile, su ámbito de vigencia es limitado a la categoría de trabajadores migratorios. Debe añadirse que esta convención no ha sido masivamente ratificada, siendo en su mayoría firmantes sudamericanos y africanos los que permitieron la entrada en vigencia de la convención recién después de 13 años de su firma ${ }^{13}$.

Una conexión entre el sufragio universal e igualitario (con base en una convención internacional de vocación universal) y el voto en el extranjero requiere, por tanto, una argumentación que posibilite imputar el segundo al primero, como parte imprescindible de su disfrute. Sin embargo, esa construcción no es posible basándose en el Pacto Internacional de Derechos Civiles y Políticos. Pese a que en su artículo 25 pareciera soportar un derecho a votar desde el extranjero al sostener:

Todos los ciudadanos gozarán, [...] y sin restricciones indebidas, de los siguientes derechos y oportunidades: [...] b) Votar y ser elegidos en elecciones periódicas, auténticas, realizadas por sufragio universal e igual y por voto secreto que garantice la libre expresión de la voluntad de los electores;

En su artículo 2 limita el ámbito territorial del deber del Estado para con la garantía del derecho a sufragio de la siguiente manera:

Cada uno de los Estados Partes en el presente Pacto se compromete a respetar y a garantizar a todos los individuos que se encuentren en su territorio y estén sujetos a su jurisdicción los derechos reconocidos en el presente [...].

Debe concluirse al menos, con los antecedentes expuestos, que no existe un indubitado derecho general fundado en las convenciones internacionales a votar desde el extranjero. El derecho que existe tiene como titulares al grupo particular de los trabajadores migratorios y en dicha regulación se establece (inc. $2^{\circ}$ art. 41 ) un amplio margen de discrecionalidad para la regulación por parte del país de origen de la forma y las condiciones en que dichos derechos podrán ser ejercidos.

Consideradas las dos ideas que se prestan más comúnmente para abogar por el voto desde el extranjero sin reflexionar sobre sus implicaciones para la teoría democrática, se debe concluir que preguntarse por quiénes deben votar no es algo que está ya respondido con la invocación de la democracia y los derechos humanos, sino algo que debe responderse y justificarse con buenas razones.

${ }^{13}$ Sobre el particular puede consultarse la información suministrada por el Comité sobre Trabajadores Migrantes de la Oficina del Alto Comisionado de las Naciones Unidas para los Derechos Humanos, en http:// www2.ohchr.org/english/bodies/cmw/. 


\section{Democracia y ciudadanía}

El derecho a voto reside en el corazón de la comprensión democrática de la ciudadanía y es uno de los elementos que permite hablar de la ciudadanía como un estatus de miembro pleno de una comunidad de iguales que se autogobiernan. Eso es cierto, en la medida que el voto permite participar directa o indirectamente en las decisiones que son tomadas por los órganos representativos del Estado ${ }^{14}$. Sin embargo, este tipo de aproximación es demasiado amplia y no responde a la pregunta sobre quiénes deben ser ciudadanos o quiénes deben votar.

La respuesta tiene que ser construida como una interpretación del principio democrático del autogobierno, conforme al cual gobernantes y gobernados deben coincidir en identidad $^{15}$. Su fundamento tiene lugar en la comprensión de todos los miembros de la comunidad como políticamente iguales ${ }^{16}$. Por supuesto que este objetivo no puede ser alcanzado hoy en día exigiendo a todos los ciudadanos que desempeñen directamente las labores del gobierno y la administración del Estado, como sucedía en la Atenas de Solón. La forma representativa del autogobierno implica una intervención ciudadana más acotada, en la que los ciudadanos se ven involucrados formalmente en la presentación y elección de los candidatos e informalmente a través de la acción política en concierto que es generada en la esfera pública y en base a la cual el gobierno puede sustancialmente seguir la voluntad de los ciudadanos. En esos términos lo que el autogobierno significa en el modelo de la democracia representativa, es en la elección de un gobierno por los ciudadanos, que con base en el principio de igualdad política deben tomar sus decisiones conforme a la regla de la mayoría ${ }^{17}$. Esta es la única forma de entender la democracia representativa como el autogobierno colectivo.

Para determinar quiénes deben tener acceso a los derechos de participación en una democracia existen varias propuestas, todas las que pasan necesariamente por contestar que todos deben tener acceso. Por supuesto que es una respuesta vaga. La pregunta se replanteará en ¿quiénes son todos? Robert Dahl responde a esta pregunta, introduciendo su principio de la inclusión con más precisión, de la siguiente forma: la categoría de ciudadanos en un Estado gobernado democráticamente "debe incluir a toda persona sujeta a la ley del Estado con excepción de los transeúntes y las personas que se ha probado son incapaces de cuidar de sí mismas" ${ }^{18}$. A esta conclusión llega a través del siguiente razonamiento: (i) los intereses de cada persona deben ser tomados en cuenta por igual a la hora de crear la ley; (ii) a menos que haya una evidencia incontrarrestable, cada

\footnotetext{
${ }^{14}$ Bauböck, op. cit., n. 4, p. 2393.

${ }^{15}$ Schmitt, Constitutional Theory, Durham, N.C: Duke University Press, 2008, pp. 255 y ss.

${ }^{16}$ Dahl, R., Democracy and its Critics, New Haven, Yale University Press, 1989, pp. 83 y ss.

${ }^{17}$ Dahl, op. cit., n. 16, pp. 89-105; 135-52. Sobre cómo la regla de la mayoría viene exigida por el principio de la igualdad política, véase Böckenförde, E. W., "La democracia como principio constitucional", en Estudios sobre el estado de derecho y la democracia, Madrid, Ed. Trotta, 2002, pp. 92 y ss.

${ }^{18}$ López-Guerra, C., "Should Expatriates Vote?", en Journal of Political Philosophy, Vol. 13, n. 2, 2005, p. 219; Dahl, op. cit., n. 16, pp. 119-31.
} 
persona debe ser considerada como el mejor juez de sus propios intereses y bienestar; por lo tanto, (iii) debe ser asumido que todos los adultos están capacitados para participar en el proceso de toma de decisiones ${ }^{19}$. Si se sigue este principio de la inclusión, la conclusión a la que debe llegarse es que todos los integrantes de la comunidad política que se encuentran bajo la sujeción del Estado deben poder participar.

Esta es una respuesta que responde a la pregunta sobre quiénes deben tener voto dentro de las fronteras jurisdiccionales de un Estado que pretende gobernarse democráticamente. Pero no responde, o al menos no lo hace directamente, a la pregunta sobre la participación de los expatriados. El principio de la inclusión puede servir de eje interpretativo de las respuestas sobre el voto desde el extranjero, en la medida que las propuestas deben ser armónicas con él y basarse en una misma lógica inclusiva. Sin embargo, como se verá, cuando se piensa en el caso de los expatriados el principio de la inclusión no es conclusivamente un principio básico y éste debe ser comparado con otras concepciones sobre la inclusión en el universo de electores.

Para responder a esta subsistente duda Rainer Bauböck sostiene que hay cuatro principales posiciones filosóficas en competencia a la hora de explicar quién debe tener el derecho a voto en una democracia ${ }^{20}$.

La primera es la posición republicana, que rechaza la posibilidad de que los ciudadanos en el extranjero voten, en la medida que sólo aquellos que estando presentes y desempeñando su rol ciudadano en la vida política pueden participar en el proceso de autogobernarse. De otra manera, la comunidad estaría parcialmente heterogobernada. Esta posición adicionalmente defiende la idea de que la propia comunidad sea la que fije los límites de su pertenencia, entregando a la propia comunidad la decisión de determinar quiénes serán los ciudadanos y qué derechos tendrán.

Una segunda posición puede ser denominada nacionalista étnica. Para sus sostenedores los ciudadanos en el extranjero siguen perteneciendo al ethos de una comunidad de cultura, descendencia y destino común que tiene el derecho a autodeterminarse, por lo que deben conservar sus derechos a participar en ella. Esta posición no parece poder conciliarse con el principio de la inclusión más arriba sugerido, en la medida que, de manera opuesta, requeriría la exclusión de aquellos que pese a tener residencia permanente e indefinida en el territorio del país no tienen los mismos antecedentes étnicos que el resto de la población ${ }^{21}$.

La posición liberal es considerada por Bauböck en dos versiones separadas. La posición de la inclusión territorial (tercera teoría) consiste en considerar como una comunidad política democrática a un conjunto de individuos sujetos a una misma autoridad política y que tienen asimismo iguales derechos a participar en el proceso de creación de las leyes. Desde esta perspectiva, la sujeción a la ley es el elemento esencial de la atribución de derechos políticos. En ese entendido, los expatriados que, por ser tales, no se ven sometidos a las

${ }^{19}$ López-Guerra, op. cit., n. 18, p. 219.

${ }^{20}$ Bauböck, op. cit., n. 2, pp. 685-6.

${ }^{21}$ Sobre este asunto, que es de una complejidad que impide su tratamiento en este trabajo, puede referirse a su análisis en Miller, D., Citizenship and National Identity, Cambridge, Polity Press, 2000. 
leyes de su país de origen, perderían el título que los habilita a participar en el proceso democrático. Pero, al igual que la posición republicana, la conclusión respecto de ellos es más drástica, de manera que deben ser excluidos. Esto tiene su fundamento en que su participación afectaría la base igualitaria del proceso democrático y determinaría los resultados de un proceso democrático cuyos efectos alcanzarán sólo a los ciudadanos residentes. En ese entendido, el argumento liberal de la no disponibilidad de la propia libertad (prohibición de la esclavitud voluntaria), para justificar una prohibición de la extensión del sufragio a los expatriados, es aplicable al caso ${ }^{22}$.

Finalmente, la cuarta teoría es la posición liberal del interés afectado. Conforme a esta teoría, todo aquel que se ve afectado por las decisiones de un Estado debe poder participar en el proceso de la toma de dichas decisiones. Desde esta perspectiva, los expatriados podrían tener derecho a voto, considerando que es probable que sus intereses se vean afectados por decisiones generadas en su país de origen. Los principales problemas con esta teoría son que es demasiado vaga, dado que es difícil concluir cuándo un individuo se verá afectado, o si personas afectadas en diversos grados deberían tener igual tratamiento. Su implementación lleva a conclusiones contraintuitivas como la de que muchos no ciudadanos y ni siquiera residentes en el país respectivo podrían tener derecho a participar en el proceso democrático interno de un Estado con el que no se encuentran directamente vinculados ${ }^{23}$. Sin embargo, esta teoría tiene la virtud de la flexibilidad. En primer lugar, permite generar criterios de atribución reflexivos a ideas adicionales que pueden estar basadas en sensibles realidades particulares. Adicionalmente, permite generar una solución intermedia que puede servir de punto de encuentro entre posiciones que tienen aspiraciones más radicales, como la republicana y la nacionalista.

\section{Reglas generales y excepciones}

Como conclusión de la sección anterior, puede afirmarse que dependiendo de la adopción de una u otra de las teorías, la respuesta a la pregunta sobre la atribución del voto varía considerablemente. A continuación se examinan dichas hipotéticas respuestas.

Tanto la teoría republicana como la teoría de la inclusión territorial consideran que el voto desde el extranjero es necesario para aquellos ciudadanos residentes pero que se encuentran transitoriamente fuera del país. Sin embargo, ambas consideran que la atribución del voto a los expatriados debe estar prohibida por el principio democrático del autogobierno. Con este sustento, puede proponerse el primer modelo de configuración del voto en el extranjero, respondiendo a las preguntas más arriba planteadas de la siguiente forma: (1) sí, debe existir un sistema de sufragio desde el extranjero que permita a ciudadanos residentes votar pese a estar ausentes el día de la elección; y (2) los expatriados no deben tener derecho a votar.

${ }^{22}$ López-Guerra, op. cit., n. 18, pp. 219-22, 225-6; Rubio-Marin, R., "Transnational politics and the democratic nation-state: normative challenges of expatriate voting and nationality retention of emigrants", en New York University Law Review, Vol. 81, 2006, p. 141.

${ }^{23}$ López-Guerra, op. cit., n. 18, pp. 222-5. 
La posición nacionalista étnica plantea con claridad la necesidad de la atribución de iguales derechos a aquellos nacionales que se encuentran en el extranjero, entre ellos el derecho a voto, sin importar el tiempo y motivo de su calidad de expatriados. Responde por tanto a las preguntas, afirmando: (1) sí, es necesario que exista un sistema de sufragio en el extranjero, que permita garantizar (2) a todos los nacionales que no se encuentren en el territorio del país ejercer el derecho a participar en la autodeterminación colectiva de la comunidad nacional a la que pertenecen.

Finalmente, para la teoría del interés afectado el otorgamiento del derecho a voto para los expatriados no parece ser una cuestión de todo o nada y dependiendo de las circunstancias contextuales que hacen operativo el principio de la afectación del interés. En ese entendido, para esta teoría (1) es necesario un sistema de votación desde el extranjero, al menos para los no expatriados y (2) la atribución del derecho a voto de los expatriados dependerá del juicio sobre la afectación de sus intereses. Esto suscita dos interesantes comentarios adicionales. El primero es que, en esta perspectiva, el sufragio de los expatriados no estaría prohibido por razones de principio como en la posición republicana, pero tampoco estaría exigido por razones de principio de una forma categórica como en la posición nacionalista, por lo que puede decirse que desde esta posición el voto de los expatriados es algo cuya discusión y adopción el Estado está autorizado a evaluar. Segundo, y conectado con la idea anterior, no parece haber razones para afirmar que este juicio deba ser realizado por otro sujeto distinto a las autoridades democráticas.

En resumen, todas las posiciones analizadas se manifiestan en favor de la conformación de un sistema de voto desde el extranjero, sin embargo responden de manera diferente la pregunta acerca de quiénes deberían poder utilizarlo. Con todo, estas respuestas se basan en una hipótesis simple que considera exclusivamente el factor fáctico de la residencia. Podrían ser denominadas soluciones generales al problema. Sin embargo, dichas respuestas generales pueden dar cabida a excepciones cuando se consideran factores adicionales. Siguiendo la historia y la regulación comparada del voto desde el extranjero se puede hablar de tres grupos de situaciones excepcionales.

La primera se refiere a los ciudadanos que residen permanentemente en el extranjero cumpliendo un servicio para el Estado. En este supuesto caben los casos paradigmáticos de militares y funcionarios diplomáticos y consulares. La segunda excepción, especialmente relevante para el caso chileno, es la que se refiere a aquellas personas que han sido exiliadas o que han sido forzadas a dejar su país por motivos de una persecución política, religiosa o racial. Por último, la tercera categoría que es tildada de especial por la literatura comparada es la de aquellos ciudadanos que se encuentran residiendo en el extranjero únicamente por razones laborales ${ }^{24}$.

Mientras la primera categoría tiende a generar acuerdo entre todas las teorías en cuanto debe serles concedido el voto desde el extranjero, la comparación entre las dos circunstancias siguientes puede ser ilustrativa de sus desacuerdos.

${ }^{24}$ Navarro et al., op. cit., n. 10, pp. 18 y ss. 
El caso de los exiliados o refugiados está marcado por el acto que da nacimiento a su calidad de tales. Fueron obligados a dejar su país de origen por la amenaza de la encarcelación, la tortura o el exterminio por una fuerza de carácter irresistible (típicamente el Estado). Lo que estaba en juego eran sus vidas, su indemnidad física y psíquica y su libertad, lo que hizo que se encontrasen, aplicando la terminología del derecho penal, en un estado de necesidad.

En el caso de los trabajadores migrantes, por otro lado, las circunstancias que dieron surgimiento a su estatuto de expatriados no son calificadas de excepcionales, dado que son el fruto de la voluntad de dejar su país de origen y asentarse en un país diferente, que es soportada por el interés racional de mejorar sus condiciones de vida y las de su familia en un país que ofrece más o mejores oportunidades laborales o mejores prestaciones sociales o, lo que es más común, ambas conjuntamente.

Si se comparan las dos situaciones, debe concluirse que su principal diferencia está en el hecho de que en el primer caso la decisión de volverse un expatriado no fue voluntaria, a diferencia del segundo caso, en el que fue la solución a una ponderación de intereses. Así consideradas, las situaciones deberían recibir un tratamiento distinto. Sin embargo, hay casos en que las características de una y otra categoría no son claramente distinguibles. En primer lugar, muchas veces las circunstancias socioeconómicas del país de origen son tan desastrosas que hacen que el fundamento voluntarista de la diferenciación se vuelva una idea vacía. En segundo lugar, una vez que las circunstancias que motivaron el exilio cesan los desterrados quedan en una situación en que deben realizar una ponderación de intereses entre volver a su patria o quedarse en el país anfitrión, lo que radica su diferencia con los trabajadores migrantes únicamente en el hecho antecedente de su exilio y no en su subsistencia. Un exiliado para quien volver a su país se le hace demasiado costoso en términos de intereses familiares, laborales y económicos, no se encuentra demasiado lejos bajo esta distinción de un trabajador migrante.

Conforme a las ideas de la teoría republicana, el trabajador migrante es una persona que toma la decisión de no compartir el destino que lo vincula a su comunidad, es más, decide dejar su comunidad por una comunidad más rica o más próspera, evadiendo así el desafío político de perseguir la justicia social de forma compartida con sus pares. Para la teoría de la inclusión territorial, asimismo, el alejamiento voluntario del expatriado comprende el dejar de ser súbdito del Estado, y por lo mismo dejar de tener la legitimidad para gobernarse a sí mismo. Finalmente, la teoría del interés es la que ofrece razones para justificar la mantención del trabajador migrante como elector.

Por otro lado, la categoría de exiliado o refugiado en cuanto generada por un estado de excepción debe ser objeto de atención más detallada. Para ello, deben considerarse tanto si el peligro que dio origen al exilio actualmente se mantiene, y de no ser así, cuánto tiempo ha pasado desde su cese. La categoría de exiliados puede dar paso a tratamientos especiales, basados en la importancia del restablecimiento del vínculo con los exiliados, que pueden incluir reparaciones y tratamientos diferenciados al resto de la población, como es el caso de la autorización para votar desde el extranjero. Las teorías revisadas no tienen una solución prediseñada para el caso del exilio. Lo que sí debe advertirse es que una medida excepcional siempre entrará en conflicto con los principios sobre los que se 
basan las reglas generales. Así, desde la teoría republicana, por ejemplo, la prolongación indefinida de la autorización para votar desde el extranjero estará afectando el principio del autogobierno colectivo.

Pueden ofrecerse ciertas conclusiones preliminares señalando que, como suele pasar con este tipo de problemas, la discusión tiende a diluirse en desacuerdos acerca de comprensiones teóricas abstractas de la identidad de la comunidad donde se vive y la justicia de las relaciones entre esa comunidad y los individuos. Estas comprensiones llevan aparejadas inclinaciones políticas que no son fáciles de reconciliar tampoco en el terreno de la política institucionalizada. Nacionalistas y republicanos, por ejemplo, parten de premisas considerablemente diversas acerca de qué constituye a la comunidad de pertenencia. Sin embargo, eso necesariamente lleva a concluir que nada puede ser dicho cuando esas teorías comprehensivas son invocadas y que ellas pueden servir de refugio contra cualquier argumento en contrario. El contenido de esta sección del trabajo tiene la función de servir como un esquema para racionalizar las posiciones que pueden encontrarse en el debate sobre la materia.

Frente a la pregunta sobre cuál teoría debe abrazarse o qué sistema de reglas debe construirse, la única respuesta que puede darse aquí pasa por la necesidad de mirar tanto al pasado como al futuro. El pasado debe ser examinado porque la respuesta a esta coyuntura requiere encontrar una reforma que tenga sentido dentro de nuestra tradición democrática. Sólo por dar un ejemplo, el nacionalismo étnico nunca ha sido parte de nuestra identidad como comunidad política, para lo que basta mirar la preeminencia del ius soli sobre el ius sanguini en las reglas sobre adquisición de la nacionalidad (artículo 10 de la Constitución Política), y por eso no tiene sentido la construcción de un modelo del voto desde el extranjero basado en sus principios. Pero para considerar correctamente el problema, también debe mirarse al futuro, teniendo claro cuál es la dirección que queremos que tome nuestra tradición democrática, considerando los excepcionales hechos de terror vinculados a la persecución política y al extrañamiento, que necesariamente deben redefinir nuestra tradición. En este punto, no puede dejar de considerarse que Chile se encuentra en una encrucijada, entre seguir perfeccionando un sistema de vida política individualista, que inspiró la Constitución de Pinochet y que ha guiado varios de los cambios que se han hecho a ella desde el retorno a la democracia, o reencontrar, como parece estar sugiriendo insistentemente la sociedad civil, un camino hacia la generación de espacios que posibiliten una vida política con acento en el autogobierno colectivo. Ésta, sin embargo, es una respuesta que no tiene sentido anticipar. 


\section{El PROYECTO DE REFORMA DEL VOTO EN EL EXTRANJERO}

El proyecto que el pasado tres de mayo de 2011 fuera rechazado en el Senado (Boletín 7335-07) presentaba características interesantes de analizar a la luz del marco de referencia hasta ahora establecido ${ }^{25}$.

Conforme a su fundamentación, lo que el proyecto pretendía hacer era posibilitar una nueva modalidad de sufragio, dirigida a aquellos ciudadanos chilenos que actualmente gozan de derecho a sufragio (conforme al art. 13 de la Constitución), pero para quienes es imposible ejercerlo dado que se encuentran fuera del país. En consecuencia, y

con el objeto de revitalizar la democracia, [el proyecto] quiere cubrir ese vacío legal, permitiendo a los ciudadanos que residen fuera del territorio nacional participar en igualdad de condiciones con los residentes del país, en procesos eleccionarios, siempre sujetos a ciertas condiciones (p. 4).

Para cumplir esa finalidad el proyecto propuso una modificación constitucional, tendiente a entregar al legislador la tarea de determinar los casos y los requisitos bajo los cuales el sufragio desde el extranjero sería factible. Para efectos de articular esos requisitos, el proyecto establecía que ellos deben dar cuenta de cierto parámetro de vinculación de los ciudadanos residentes en el exterior con Chile, cuya fijación también sería entregada a la competencia del legislador ${ }^{26}$.

El proyecto ofrece dos puntos de interés para ser discutidos en el contexto de este trabajo. El primero es la necesidad de la reforma constitucional para la introducción de esta legislación. El segundo consiste en la perseverancia del gobierno en la exigencia de una vinculación con Chile para poder ejercer el derecho a voto desde el extranjero.

En relación al primer punto, la observación del derecho comparado evidencia que el sufragio desde el extranjero puede estar consagrado en la Constitución, en la legislación o en regulación administrativa. El primer caso es bastante raro, y la mayoría de los países incorporan estas disposiciones en la legislación electoral, pese a que gran número de detalles regularmente quedan entregados a normas administrativas ${ }^{27}$.

El proyecto pretendía reformar la Constitución y delegar a la legislación la regulación de las formas y casos en que la participación desde el extranjero estaría permitida y las circunstancias que serían constitutivas de un vínculo suficiente con Chile para

${ }^{25}$ El proyecto de ley rechazado tiene un contenido similar al proyecto del boletín 6950-07 que todavía permanece en tramitación y mediante el cual la iniciativa podría se repuesta en la discusión pública.

26 El principal artículo del proyecto de ley de reforma establecía: "Artículo $1^{\circ}$.- Introdúzcase un nuevo inciso cuarto al artículo 13 de la Constitución Política de la República: "Los ciudadanos con derecho a sufragio que se encuentren fuera del país y mantengan vínculos con Chile podrán sufragar desde el extranjero en las elecciones y plebiscitos que establezca una ley orgánica constitucional. Dicha ley establecerá también las condiciones y formas en que se ejercerá este derecho y regulará las circunstancias que constituyen tal vinculación"”.

${ }^{27}$ España (art. 68.5) y Portugal (art. 172) son los casos más llamativos de consagración constitucional de la institución, Nohlen y Grotz, op. cit., n. 7. 
autorizar el voto. En opinión de Rodrigo Correa, el proyecto pretendía establecer reformas innecesarias:

Para permitir votar a los ciudadanos desde el extranjero no se requiere modificar la Constitución Política. Ésta reconoce a todos los ciudadanos el derecho de sufragio, sin calificación alguna. Son las leyes sobre sistema de inscripciones electorales y servicio electoral y sobre votaciones populares y escrutinios las que han diseñado un sistema electoral que no contempla la posibilidad de votar desde el extranjero. Para permitir dicho voto, bastaría con modificar dichas leyes ${ }^{28}$.

Este punto se hace evidente cuando aparece, como ya se observó, que en el derecho comparado el voto en el extranjero es usualmente una cuestión meramente legislativa. Si la reforma de la Constitución no era requerida, en la medida que la actual regulación constitucional no impide el voto desde el extranjero, cuáles son las razones para tal reforma es una cuestión que el proyecto no responde. Y es que no existe una argumentación para ello que no caiga necesariamente en una contradicción. Si bien se puede entender que lo que se lleva a cabo con la reforma es una cuestión sustantivamente constitucional dado que implica la ampliación del universo de ciudadanos, algo que desde el punto de vista de la teoría constitucional democrática requiere un proceso de reflexión del más alto nivel, dicha reflexión choca de frente con la explícita atribución de ciudadanía sin reparo a la residencia de sus titulares que la Constitución chilena hace. En otras palabras, si los ciudadanos que viven en el extranjero poseen el derecho a sufragio, dado que la Constitución no hace distinción alguna (al menos no a este respecto) entre ciudadanos de diversas clases, no puede partirse de la base de que en esta reforma lo que se está haciendo es algo tal como la ampliación de la ciudadanía o extendiendo el derecho a sufragio a quienes anteriormente no contaban con él. La extensión de la ciudadanía es un asunto constitucional, pero no es sobre la extensión de la ciudadanía (sobre la cuestión de la titularidad) sobre lo que la implementación del voto en el extranjero está tratando, sino sobre la mera cuestión del procedimiento.

Hecha la aclaración desde el punto de vista de la regulación, vale la pena considerar el problema desde una perspectiva teórica. Si existe reticencia a aceptar que personas no vinculadas a Chile puedan tener influencia en el contenido de las decisiones políticas que son tomadas en el país, la verdadera pregunta parece ser si tiene sentido que esas personas sean ciudadanos. Así reformulada la cuestión central de la discusión, la reforma constitucional que debería ser intentada en este contexto debería referirse a las causales de pérdida de la ciudadanía o suspensión de la ciudadanía (en lo que se ha llamado una

${ }^{28}$ Correa, R., "Votar desde el extranjero", en El Post, 2011. [http://elpost.cl/content/votar-desdeel-extranjero], acceso en 24.05.2011. En el mismo sentido, Núñez, J.I., "¿Soluciones? al voto chileno en el exterior. Un Caballo de Troya”, en Diario Constitucional, 2011. [http://www.diarioconstitucional.cl/ mostrararticulo.php?id=39], acceso en 02.08.2011; y Fernández, M., "Que todos voten", en La Tercera 12/04/2011 [http://www.tercera.com/noticia/opinion/ideas-y-debates/2011/04/895-357685-9-que-todosvoten.shtml], acceso en 05.06.2011. 
ciudadanía dormida) ${ }^{29}$ para aquellos chilenos residentes en el extranjero que no cumplan con determinados requisitos. Otra alternativa es la redefinición del universo de ciudadanos con base en la reducción del universo de nacionales. Como lo ha sugerido Rodrigo Correa, la Constitución amplió desmesuradamente el reconocimiento de la nacionalidad en la reforma del año 2005 hacia personas que efectivamente carecen de vínculo con Chile, eliminando la causal de pérdida de nacionalidad por nacionalización en el extranjero y ampliando la nacionalización por ius sanguini hasta la segunda generación ${ }^{30}$. Esta reflexión sirve para introducir el segundo de los puntos de interés que el proyecto de reforma plantea.

Sobre la exigencia de la necesaria vinculación con Chile para la atribución del voto a los expatriados, el proyecto menciona:

[...] ésta se encuentra íntimamente ligada con la función política que implica el pertenecer al cuerpo electoral de un país. Si un chileno residente en el extranjero se encuentra aún vinculado a su país, éste naturalmente siente preocupación por los destinos de Chile y, por ende, puede asumir plenamente la responsabilidad que implica el participar de una elección (p. 5).

La vinculación con el país fue uno de los puntos que hizo que el proyecto no lograra el quórum exigido en el Senado. Bajo la actual regulación constitucional, se ha sostenido que el proyecto sería inconstitucional, dado que estaría discriminando entre dos clases de ciudadanos para la atribución del derecho fundamental de sufragio: los suficientemente vinculados a Chile y los que no cumplen con los requisitos de vinculación ${ }^{31}$. Sería interesante observar cómo sería resuelta la antinomia entre el permiso para discriminar contenido en el proyecto de reforma con la prohibición de discriminar contenida tanto en el derecho a la igualdad como en la atribución universal del derecho a sufragio ${ }^{32}$.

En el derecho comparado es común ver que los sistemas de votación desde el extranjero distinguen entre expatriados basándose en diversas categorías, principalmente la ya señalada entre ciudadanos residentes y expatriados. Tal distinción es posible si se rediseñan las normas constitucionales adecuadamente. Sin embargo, nuevamente desde un punto de vista teórico la duda que subsiste, y para la cual la primera parte de este trabajo ha intentado configurar un marco conceptual, es cuál es el tipo de vinculación que se requiere y cuáles pueden ser las circunstancias que lo demuestren.

\footnotetext{
${ }^{29}$ Rubio-Marin, op. cit., n. 22, p. 139.

${ }^{30}$ Correa, op. cit., n. 28.

31 Correa, op. cit., n. 28.
}

32 Este caso desencadenaría la fútil discusión teórica sobre el problema de la inconstitucionalidad material de una ley de reforma a la Constitución, pero, y más interesante en la práctica, generaría la pregunta sobre la infracción a las obligaciones internacionales que vinculan al Estado chileno. 


\section{HaCia un Sistema de voto Desde El EXTRANJero}

En la primera sección se presentaron algunos argumentos teóricos para la mejor comprensión del voto desde el extranjero. Ello se hizo desde un punto de vista que podría llamarse teórico, considerando especialmente la importancia de la democracia en la atribución de los derechos de ciudadanía. Sin embargo, después de presentar los términos del proyecto en la segunda sección, lo que en esta última sección se pretende realizar es un análisis que considera más seriamente el contexto institucional y las posiciones y definiciones que un modelo de votación desde el extranjero requiere contener. Hay gran variación entre las diferentes legislaciones comparadas que permiten el voto desde el extranjero. En particular, son diferentes los grupos que tienen permitido votar, las circunstancias en las que deben encontrarse para efectuar el sufragio, las votaciones en las que éste es permitido, la forma de la votación y la manera en que dichos votos serán contabilizados ${ }^{33}$. Partiendo de esta base, ninguna regulación puede ser ingenua a las implicaciones que ella acarrea ni menos a los propósitos a los que se debe. En ese entendido, se revisarán las variantes que puede adoptar un sistema de votación desde el extranjero con especial consideración de las particularidades del sistema jurídico chileno y de las circunstancias extralegales que han hecho que la demanda por el voto de los expatriados sea especialmente sensible en nuestro país.

En lo que sigue, se discutirá, en primer término, el caso de las restricciones que se aplican para ejercer el voto desde el extranjero, en particular la restricción del tiempo de residencia en el extranjero. En segundo lugar, se revisará brevemente en cuáles de las posibles elecciones el sufragio desde el extranjero es posible. Luego se considerará el particular caso de los exiliados, lo que requiere, como se ha señalado, un análisis distinto del utilizado para el supuesto general. Finalmente, una breve consideración de los problemas de la implementación del sistema será presentada.

\section{Restricciones o condiciones que se aplican al voto desde el extranjero}

Si bien hay algunos países que conceden el voto desde el extranjero sin restricciones, la mayoría de ellos consideran ciertas limitaciones. Las más comunes son la de no haber excedido determinado número de años viviendo en el exterior del país de origen y la de inscribirse en un registro especial al efecto. Sin embargo, hay otras tales como llevar un determinado período de tiempo viviendo el país de destino desde el cual se pretende sufragar o que se cumpla con un determinado número mínimo de ciudadanos que quieran sufragar en el país de destino ${ }^{34}$.

De entre esos requisitos, el más interesante de considerar es el de llevar un determinado número de años viviendo en el extranjero. Ello dado que es un requisito que es utilizado para evaluar la vinculación del ciudadano con el Estado de origen. Así, cuando

\footnotetext{
33 Braun et al., op. cit., n. 1, p. 3.

${ }^{34}$ Braun et al., op. cit., n. 1, p. 6.
} 
un sujeto traspasa el número de años establecido, el sistema jurídico presume que la vinculación necesaria con el Estado de origen se ha disuelto o al menos se ha debilitado, eliminando el fundamento de la atribución de los derechos políticos asociados a la calidad de ciudadano. La regla de la necesidad de inscripción especial no sería aplicable a Chile, dado que la Constitución recientemente fue modificada para acoger un sistema de inscripción automática (artículo 18 inciso $2^{\circ}$ ).

Ya se ha discutido que un proyecto que intente entregar derecho a sufragio sólo a ciertos ciudadanos se enfrenta al problema de la prohibición de discriminar entre ciudadanos. Pero si se considera el problema desde la perspectiva de lege ferenda (más arriba se sugirieron algunas soluciones para abordarlo), es permisible discutir la legitimidad del requisito de permanencia en el extranjero en sus propios términos. El requisito de permanencia es la forma de implementar, de una forma más flexible que la sola negativa, las perspectivas teóricas sobre el voto desde el extranjero revisadas más arriba. El límite máximo de permanencia, en otros términos, es el reverso del requisito de permanencia en el territorio del país de origen, al que las perspectivas teóricas asignan tanta importancia.

Este requisito puede ser teóricamente criticado, desde la perspectiva nacionalista étnica, porque estaría denegando la posibilidad de participar a personas que siguen siendo parte de la nación. Para las demás teorías la regla del plazo permite implementar el principio de la autodeterminación democrática y la exclusión de alguien que comienza a configurarse como un miembro de otra comunidad.

Pero incluso si se considera que las razones nacionalistas no son atendibles, puede ser sostenido como arbitrario el hecho de considerar un plazo cualquiera para juzgar la desvinculación de un ciudadano con su país de origen. La respuesta pasa por entender que la función de los plazos es eliminar complejidad y estabilizar expectativas, y en ese sentido todo plazo tiene un cierto contenido de arbitrariedad si pretende cumplir dicha función. Si tiene que proponerse un plazo, es sensato mirar los plazos que la propia Constitución consagra para fines similares. Si la Constitución considera que cinco años residiendo en el país es un tiempo suficientemente extenso para concluir la vinculación permanente de un extranjero con Chile (atribuyéndole derecho a sufragio), ese lapso de tiempo parece ser el razonable para aplicar una regla de sufragio en el extranjero. Si el ciudadano se encuentra residiendo en el extranjero por más de cinco años, queda excluida la posibilidad de ejercer su derecho a sufragio. Este sistema está consagrado en las legislaciones de Canadá y Australia (cinco y seis años, respectivamente). Otro elemento a tener en cuenta en la fijación de los años necesarios para esta expulsión del registro electoral podría ser el periodo de duración de los representantes elegidos en cada una de las elecciones en que los ciudadanos pueden votar. Por ejemplo, cuatro años para el caso del Presidente de la República ${ }^{35}$. 
Tipos de proceso electoral en que se puede sufragar

El voto desde el extranjero puede ser aplicado a las elecciones nacionales, ya sea parlamentaria (India y Holanda) o presidencial (casos de Brasil y Bolivia) o ambas (Argentina e Israel), a las elecciones locales (Irlanda y Estados Unidos) o referéndum (Austria y Colombia) ${ }^{36}$.

En el caso de Chile su aplicación no parece problemática en absoluto para el caso de elección del Presidente de la República y para el caso de referéndum. Sí parece difícil aplicarlo a la elección de los parlamentarios y de las autoridades locales. Diferentes razones gobiernan la solución para estos casos.

En el caso de las elecciones de parlamentarios, el actual sistema electoral que distribuye los representantes por distritos y circunscripciones plantea dos problemas para el voto desde el extranjero. El lugar de residencia dentro del país es el elemento definitorio para asignar a un elector a un particular distrito. Surge entonces la pregunta sobre a cuáles distritos y circunscripciones estarán adscritos los votantes en el extranjero y, quizás más seriamente problemático, cómo se realizará el recuento de esos votos y se adicionarán al resultado que se produzca en el territorio del país. En el derecho comparado existen representantes parlamentarios para los residentes en el extranjero. Esa es una figura que si bien soslaya las dificultades técnicas, incorpora una modificación aún más considerable en el sistema electoral.

El caso de las elecciones locales, por otro lado, son razones relacionadas con la función que dichas autoridades cumplen las que llevan a concluir que los residentes en el extranjero no tienen interés en participar en dichas elecciones. La participación en el gobierno local tiene como principal justificación la residencia en la localidad. Así lo entienden, en el derecho comparado, los Estados que confieren derechos políticos en el ámbito local a aquellos extranjeros residentes pese a no concedérselos en el ámbito nacional ${ }^{37}$.

\section{El caso de los exiliados}

Quizás la más interesante circunstancia que está envuelta en esta materia está completamente ausente en el mensaje del rechazado proyecto. Gran parte de la presión política que ha sido constantemente soportada por la izquierda para la adopción de una fórmula de voto desde el extranjero dice relación con utilizarla como una herramienta de justicia transicional, esto es, como parte de la reparación a la cual tienen derecho quienes vieron violados sus derechos humanos por la dictadura militar ${ }^{38}$.

\footnotetext{
${ }^{36}$ Navarro et al., op. cit., n. 10, pp. 15 y ss.

${ }^{37}$ Bauböck, op. cit., n. 2, p. 284.
}

38 Justicia transicional es definida por el International Center of Transitional Justice como "la respuesta a las violaciones sistemáticas o generalizadas de los derechos humanos. Busca reconocimiento para las víctimas y promoción de paz, reconciliación y democracia. La justicia transicional no es una forma especial de la justicia, sino justicia adaptada a las sociedades que están transformándose después de un período de 
El largo tiempo que ha pasado desde el éxodo al que se vieron obligados muchos chilenos, hace que de adoptarse una regla como la del plazo, ellos, uno de los principales grupos impulsores de la medida, quedarían fuera de los alcances previstos. De aplicarse una hipotética regla de, por ejemplo, cinco años, los exiliados que han tenido su residencia permanente en el extranjero por más de 25 años quedarían excluidos del padrón electoral.

Si se toman seriamente las razones para tratar a aquellos que fueron exiliados de una forma diferente al tratamiento de los expatriados en general, lo que resta es encontrar una solución excepcional que permita dar cuenta de la situación también excepcional de la que ellos fueron objeto. En ese entendido, dos son las medidas que en mayor o menor magnitud podrían favorecer el voto de los exiliados. La primera es incorporar una regla especial que conceda el derecho a votar a los exiliados por razones políticas sin consideración del número de años que hayan residido en el exterior. La segunda medida, que toma más en serio los principios que yacen detrás de la demanda de autogobierno, al tiempo que también contempla la situación del exilio como una circunstancia a ponderar, es incorporar una regla transitoria que posibilite la participación de los exiliados durante un periodo de tiempo una vez establecido el sistema, pero que esté destinada a desaparecer en el mediano plazo, atribuyendo al retorno a la patria de los exiliados la potencialidad de seguir gozando de derechos políticos.

\section{Los problemas de implementación}

Quizás la principal objeción de carácter general que enfrenta la implementación del voto desde el extranjero es el costo asociado que ella conlleva. El costo de un voto desde el extranjero excede con creces el costo de un voto producido en el interior del país. En ese entendido, cabe preguntarse si deben gastarse grandes sumas de dinero para dar la posibilidad de votar a quienes están lejos del país.

Pero las complicaciones no son solamente financieras. La implementación del voto en el extranjero es complicada técnicamente por varios factores, entre los que se cuentan el número de votantes del que hay que dar cuenta, los lugares de votación, las distancias que se ven involucradas y las complejidades que incorpora en el sistema de votación ${ }^{39}$.

Sin embargo, puede ser observado que en el derecho comparado ha surgido una importante serie de mecanismos que dan cuenta de esas dificultades estableciendo diferentes vías más o menos idóneas para realizar el sufragio desde el exterior. Entre ellas se cuentan (i) el sufragio en embajadas o consulados, o en lugares de votación especialmente habilitados; (ii) el sufragio por correo; (iii) el sufragio por mandato; y (iv) el voto electrónico ${ }^{40}$. Todos ellos tienen la virtud, no sólo de servir de soporte al procedimiento del voto desde el exterior, sino de permitir un mayor acceso a la votación

violación generalizada de los derechos humanos" (http://ictj.org/publication/what-transitional-justice. [acceso el 10/07/2011]).

39 Braun et al., op. cit., n. 1, p. 4.

${ }^{40}$ Braun et al., op. cit., n. 1, p. 7; Navarro et al., op. cit., n. 10, pp. 28 y ss. 
por parte de los ciudadanos en el exterior. Este elemento no debe ser subestimado, en cuando la participación de estos ciudadanos dependerá también del costo que signifique para ellos tener acceso a la votación ${ }^{41}$.

En este escenario de sistemas alternativos surge un nuevo foco de tensión a considerar, que consiste en un probable déficit en la satisfacción de los principios de integridad de los procedimientos democráticos de votación. Dicho de otra manera, hay una relación inversamente proporcional entre un sufragio barato y un sufragio que cumple con las garantías de autenticidad, secreto, seguridad y confiabilidad. Luego, parece ser que la respuesta basada en la proliferación de nuevas tecnologías para solucionar el problema del costo de la elección desde el exterior no es del todo obvia ${ }^{42}$.

\section{CONCLUSIÓN}

Este trabajo estuvo dirigido a la consideración de ciertos elementos, principalmente tomados del derecho comparado y de la teoría política, que pueden hacer más inteligible la discusión sobre el sufragio desde extranjero en el sistema político chileno.

La primera parte de este trabajo intentó mostrar la importancia de discutir el fundamento del voto desde el extranjero, mediante la aclaración de algunas de las premisas que subyacen a su discusión. Se contempló que hacer la distinción entre procedimiento de votación y titularidad del voto puede enriquecer y aclarar el contenido del debate. También se trató brevemente el caso de los argumentos de la democratización y los derechos humanos en favor del voto desde el extranjero y se concluyó que, en tanto estos no eran concluyentes, debía realizarse un estudio más profundo sobre la relación entre esta clase de voto y el principio de la democracia. Se revisaron, consecuentemente, cuatro teorías (republicana, nacionalista, sujeción territorial e interés afectado) que de manera general pretenden determinar quiénes y en qué circunstancias deben votar en una democracia, y se revisaron tres categorías excepcionales (personas al servicio del país, exiliados y trabajadores migrantes) que deben ser consideradas por las reglas generales sobre el voto desde el extranjero propuestas desde la teoría. Las conclusiones sustantivas de esta parte no son propositivas, sino que se limitan a ofrecer un marco conceptual que sirva de referencia a la hora de conducir la discusión en los términos que son requeridos por un debate crítico y racional.

La segunda parte del trabajo examinó el proyecto de ley de reforma constitucional que fuera rechazado en el Congreso, concluyendo que lo afectaban problemas significativos desde una perspectiva teórica. Su inconsistente jerarquía constitucional, su afectación al derecho a la igualdad y la total falta de tratamiento del caso de los exiliados, fueron el diagnóstico que una nueva introducción en la agenda parlamentaria debe considerar.

\footnotetext{
${ }^{41}$ Bauböck, op. cit., n. 4, p. 2407.

${ }^{42}$ Bauböck, op. cit., n. 4, p. 2407.
} 
Finalmente, en la tercera parte del trabajo se consideraron en concreto los elementos que una legislación sobre el voto desde el extranjero debe contemplar. En relación a los requisitos que podrían considerarse para demostrar su vinculación con el país, se sugirió que un plazo de cuatro o cinco años parecía como adecuado como límite máximo para residir en el extranjero. En relación a en qué elecciones deben participar los expatriados, se sugirió como menos problemática la sola participación en elecciones nacionales presidenciales y referéndum. Contemplando el caso de los exiliados, se apuntó hacia que la mejor solución pasaría con crear una regla especial o transitoria que los beneficie. Finalmente, para el caso del problema de implementación del sistema de advirtió que pese a la existencia de varios sistemas que lo hacen posible, el descenso del costo económico de la implementación redunda en una necesaria pérdida en la integridad de los principios de sufragio.

\section{BIBLIOGRAFÍA}

BAubÖCK, R., "Stakeholder citizenship and transnational political participation: A normative evaluation of external voting", en Fordham Law Review, Vol. 75, 2006, pp. 2393 ss.

Bauböck, R., "Expansive Citizenship-Voting beyond Territory and Membership", en Political Science and Politics, Vol. 38, no. 4, 2005, pp. 683-687.

BöCKENFÖRDE, E. W., "La democracia como principio constitucional”, en Estudios sobre el estado de derecho y la democracia, Madrid, Ed. Trotta, 2002.

Braun, N. y Gratschew, M., "Introduction" en Ellis, A., Navarro, C., Morales, I. y Wall, A. (editores), Voting from abroad: the International IDEA handbook, Stockholm, International Institute for Democracy and Electoral Assistance / The Federal Electoral Institute of Mexico, 2007.

Collyer, M. y Vathi, Z., "Patterns of Extra-territorial Voting", Working Paper T22, Sussex Centre for Migration Research, October, 2007.

Correa, R., "Votar desde el extranjero", en El Post, 2011. [http://elpost.cl/content/votar-desdeel-extranjero], acceso en 24.05.2011.

DAHL, R., Democracy and its Critics, New Haven, Yale University Press, 1989.

DAHL, R., On Political Equality, New Haven, Yale University Press, 2006.

ElLIs, A., "The history and politics of external voting", en Ellis, A., Navarro, C., Morales, I. y Wall, A. (editores), Voting from abroad: the International IDEA handbook, Stockholm, International Institute for Democracy and Electoral Assistance / The Federal Electoral Institute of Mexico, 2007.

FERnÁndeZ, M., "Que todos voten”, en La Tercera 12/04/2011 [http://www.tercera.com/noticia/ opinion/ideas-y-debates/2011/04/895-357685-9-que-todos-voten.shtml], acceso en 05.06.2011.

Hayden, G.M., "False Promise of One Person, One Vote", en Michigan Law Review, Vol. 102, 2003 , pp. 213 y ss.

López-Guerra, C., "Should Expatriates Vote?", en Journal of Political Philosophy, Vol. 13, no. 2, 2005, pp. 216-234.

Miller, D., Citizenship and National Identity, Cambridge, Polity Press, 2000.

Navarro, C., Morales, I., Gratschew, M., "External voting: a comparative overview", en Ellis, A., Navarro, C., Morales, I. y Wall, A. (editores), Voting from abroad: the International IDEA handbook, Stockholm, International Institute for Democracy and Electoral Assistance / The Federal Electoral Institute of Mexico, 2007. 
Nohlen, D. y Grotz, F., "External voting: legal framework and an overview of electoral legislation", en Boletín Mexicano de Derecho Comparado, Vol. 99, 2000, pp. 1115-1145.

NúÑEZ, J.I., “'Soluciones? al voto chileno en el exterior. Un Caballo de Troya”, en Diario Constitucional, 2011. [http://www.diarioconstitucional.cl/mostrararticulo.php?id=39], acceso en 02.08.2011.

RUbIO-MARIN, R., "Transnational politics and the democratic nation-state: normative challenges of expatriate voting and nationality retention of emigrants", en New York University Law Review, Vol. 81, 2006, pp. 117 y ss.

Schmitт, C., Constitutional Theory, Durham, N.C: Duke University Press. 
